\title{
Renal artery stenosis: is angiography still the gold standard in 2011?
}

\author{
Kjell Tullus
}

Received: 16 December 2010 /Revised: 17 December 2010 /Accepted: 22 December 2010 /Published online: 30 January 2011

(C) IPNA 2011

\begin{abstract}
Renovascular hypertension (RVH) is an unusual cause of hypertension in children that is important to diagnose as it is potentially curable with angioplasty or surgery. Digital subtraction angiography is the gold standard for diagnosing $\mathrm{RVH}$; for other methods like ultrasound, isotope studies, magnetic resonance angiography and computed tomography angiography sensitivity is still too low to reliably exclude RVH. Clinical and laboratory criteria that suggest RVH will be proposed and recommendations when digital subtraction angiography should be performed will be made. A treatment algorithm that suggests when to use medical treatment, interventional radiology and surgery in children with RVH will be given.
\end{abstract}

Keywords Renovascular hypertension · Diagnosis .

Angiography $\cdot$ Treatment $\cdot$ Angioplasty

\section{Introduction}

Renal artery stenosis (RAS) is an unusual cause of hypertension in children and most paediatric nephrologists will only see a very limited number of cases during their active professional life [1]. It is, however, very important to diagnose and treat RAS correctly as it is potentially curable with angioplasty and/or surgery.

As RAS is so relatively rare in children no larger prospective studies have been performed. I will give here recommendations on how children with suspected renovas-

\section{K. Tullus $(\bowtie)$}

Great Ormond Street Hospital for Children,

Great Ormond Street,

London WC1N 3JH, UK

e-mail: tulluk@gosh.nhs.uk cular hypertension (RVH) should be investigated and treated. This will be based on the limited literature and our experience at a large referral centre for these children.

\section{When should we suspect renovascular hypertension?}

Children with renovascular hypertension (RVH) will at presentation often have a very high blood pressure (BP); systolic BP values up to 200 or more are not uncommon [2]. Their presenting symptoms are often very severe; heart failure or cerebral symptoms like stroke, seizures or a facial palsy. Importantly, however, in about one third of children the RVH is diagnosed as an incidental finding.

The diagnosis and treatment of RVH is often delayed for a long time, in particular in children, who at onset have no symptoms. There are no clear symptoms or signs that clearly indicate RAS. We use a number of clinical and laboratory features to decide when to suspect this diagnosis (Table 1). All children who have at least one of those signs have been investigated in our centre using renal angiography. With these criteria we have a positive diagnosis of RVH in $50 \%$ of our investigations.

\section{Which imaging technique should be used?}

Ultrasound (US), isotope studies, magnetic resonance angiography (MRA) or computed tomographic angiography (CTA) are all part of the diagnostic armamentarium of RAS. The imaging should be done in a stepwise fashion. It should consider the need to investigate several vascular beds as a majority of children with RAS display involvement of multiple arteries [3-5]. At least $50 \%$ of the children have bilateral RAS; some have mainly involvement at the 
Table 1 When to suspect renovascular hypertension and perform an angiography

\section{Very high BP}

Secondary symptoms of high BP-including cerebral symptoms, cardiac failure and facial palsy

Hypertension not controlled on $\geq 2$ antihypertensive drugs

Diagnosis of a syndrome with a higher risk of vascular disease, e.g. neurofibromatosis, Williams' syndrome

Signs of vasculitis - in particular Takayasu disease

Known or suspected previous vascular insult such as renal artery thrombosis or umbilical artery catheterisation

Transplanted kidneys

Bruit heard over the renal artery or arteries

Elevated peripheral plasma renin or moderate hypokalaemia

arterial ostium, while others have RAS in smaller intrarenal arteries, both of which can be difficult to diagnose on non-invasive imaging.

In a quarter of our children the main problem was midaortic syndrome (MAS) [5]. Twenty percent of children have major involvement both extra- and intra-cerebral blood vessels. These children might need treatment of their carotid arteries or other procedures to improve the intracerebral blood flow. It is, however, not unusual for them to be left with impaired cerebral perfusion that is not amenable to further treatment. In these cases there can be a need to keep the blood pressure above normal levels to ensure sufficient cerebral blood flow. The optimal blood pressure in these children can be very difficult both to define and to achieve.

Some children have narrowing of the iliac vessels with often non-specific symptoms of claudication. The child might only be less interested in taking part in physical activities compared with their friends. Narrowing of the intestinal arteries is found in a third of the children. This part of the disease is in our experience mostly asymptomatic; we see very little abdominal angina or growth failure. The collateral formation seems here to be very efficient.

\section{Imaging}

The duplex US can in typical cases show reduced perfusion to the kidneys, narrowing of renal arteries and the aorta and an impaired perfusion index (Fig. 1) [6].

The use of pre- and post-captopril DMSA (or another isotope) was a wonderful theory where the angiotensin converting enzyme inhibitor should have reduced the perfusion pressure enough to induce uptake defects on the isotope scan that were not found on the pre-captopril study. Previous small studies have suggested that it could be

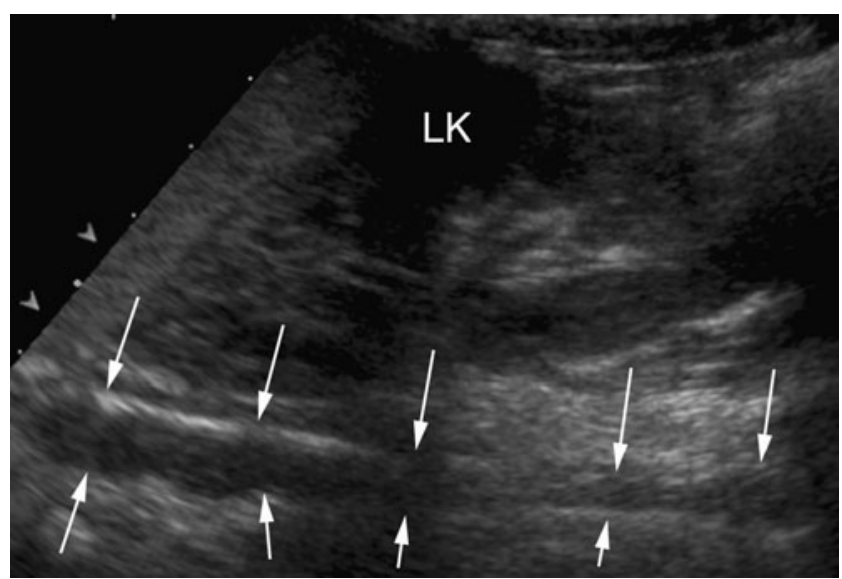

Fig. 1 Narrowed aorta in mid-aortic syndrome on ultrasound

useful as a screening test, but a recent large evaluation of the usefulness of this method from our institution did, however, find a sensitivity of only $47 \%$, which thus renders this method useless [7]. The largest and best studies in adults have found sensitivity between 72 and $81 \%$, which is also too low if a potentially curable problem is sought.

Both MRA and CTA can produce beautiful pictures of the vasculature (Figs. 2, 3). Studies in adults and case reports in children have shown that the sensitivity of these methods is 64-93\% and 64-94\% respectively (Table 2). None of these imaging techniques is sensitive enough to rule out renovascular disease with a high enough degree of certainty [8, 9]. The gold standard investigation is, thus, presently digital subtraction angiography (DSA). In medical settings where DSA is difficult to perform other methods, preferably MRA or CTA, can be used as reasonable secondline investigations after ultrasound.

Digital subtraction angiography is, however, the only method that can reliably diagnose RAS and it must therefore be done in all cases with a strong suspicion of

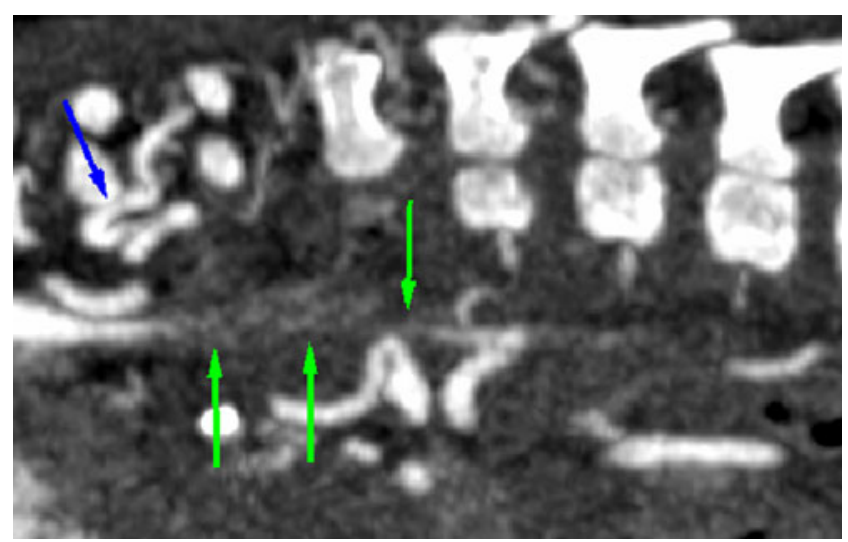

Fig. 2 Computed tomographic angiography showing narrowing of the aorta 


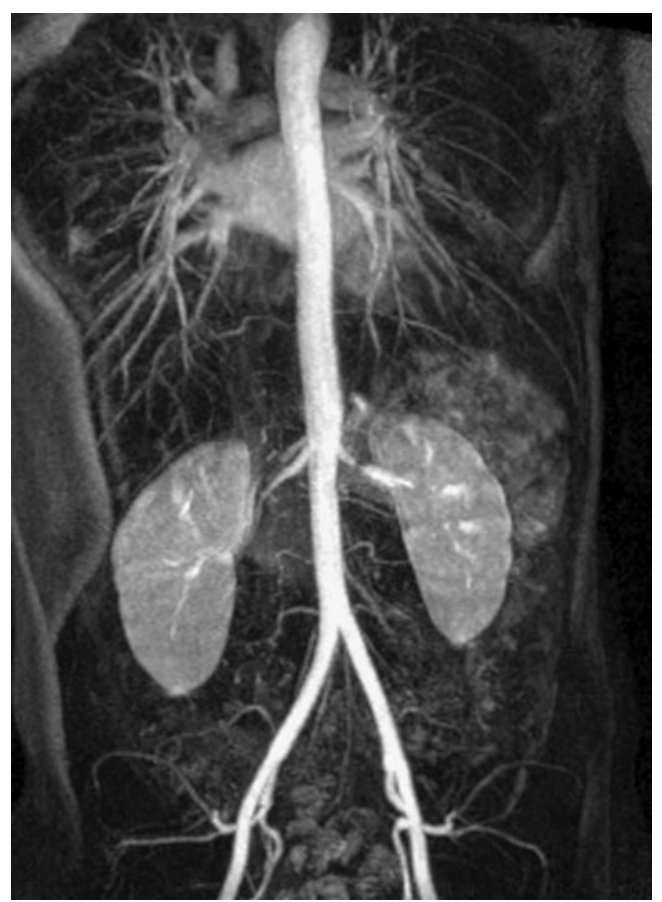

Fig. 3 Magnetic resonance angiography showing left renal artery stenosis and possible right renal artery stenosis

this disease (Fig. 4). It also has the advantage that the child can be treated during the same procedure.

\section{Angioplasty with or without stenting}

At our centre angioplasty is the most commonly used treatment for children with RVH. Angioplasty in children, in particular in the youngest age group is challenging and should always be done by an interventional radiologist who is very experienced in children. We do always have a vascular surgeon on stand-by to be ready to help if something untoward happens. Luckily enough we have not needed that help so far.

The affected arteries can be totally occluded, without any lumen seen on angiography. These arteries can sometimes still be recanalised and dilated. This needs highly specialised equipment, e.g. a so-called cutting balloon that

Table 2 Diagnostic accuracy of ultrasound and pre- and postcaptopril isotope studies

\begin{tabular}{lll}
\hline Technique & Sensitivity (\%) & Specificity (\%) \\
\hline Ultrasound & $73-85$ & $71-92$ \\
Captopril renography & $52-93$ & $63-92$ \\
CTA & $64-94$ & $62-97$ \\
MRA & $64-93$ & $72-97$ \\
\hline
\end{tabular}

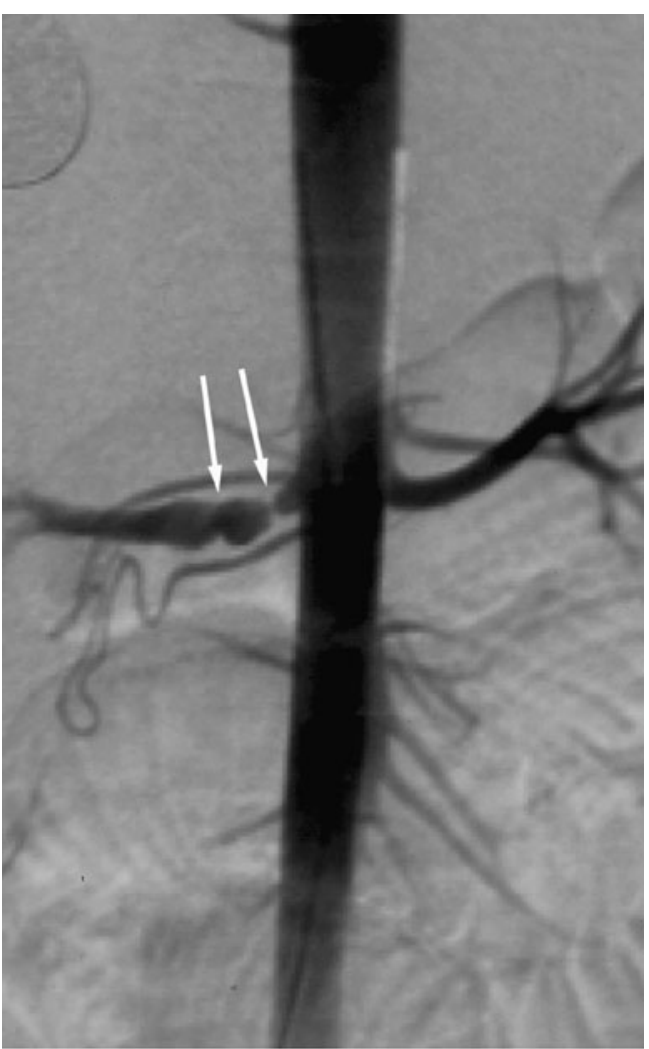

Fig. 4 Typical string of beads appearance in fibromuscular dysplasia

enables the interventional radiologist to open up blood vessels that otherwise would be impossible to treat [9].

The long-term success rate seems, at present, to be between 50 and $90 \%$ with technical failures or late stenosing events being the most important problems [3, 10-12]. The technical failures are with increasing experience and improving equipment getting less common. It is, however, important to store a large number of different catheters of different sizes to be able to undertake successful procedures in children of all ages and sizes. This is quite costly as these catheters are very expensive.

Some blood vessels that have been successfully dilated return to their original size soon after the balloon is removed. In such cases stenting provides a possibility for a successful outcome. Late re-obstruction can be caused by thrombotic events or by intimal hyperplasia. Anti-platelet treatment provides some protection against thrombosis. Development of intimal hyperplasia can partly be prevented with so-called drug eluting stents. These stents are coated with drugs like sirolimus, which very slowly elute into the lumen. Their use in adult coronary arteries is still debated and the use in children has so far been very limited [13].

A further reason for the lack of major improvement in the blood pressure despite technically good results is that a large proportion of children that are treated have involve- 
ment of several other blood vessels including the intra-renal arteries and the aorta (mid-aortic syndrome) [5, 13].

\section{Other treatments}

\section{Medical treatment}

Medicines are used initially to try to control the often very high blood pressure in the large majority of children with RVH. This will improve the blood pressure, but not enough, and other treatments will be needed. It is very important not to use drugs that work on the renin angiotensin axis, i.e. angiotensin converting enzyme inhibitors (ACEi) and angiotensin II receptor blockers (ARB), as they will impair the kidney function. This is difficult to monitor in children with unilateral disease as the changes in serum creatinine might be very small. It is not unusual to see a well functioning kidney on DMSA scintigraphy go from $50 \%$ relative function to less than $10 \%$ in a short time.

In a proportion of children interventional and/or surgical treatment will not be possible, e.g. for technical reasons. The narrowed blood vessels may be too small to be possible to treat and medical treatment can be the only option. It is, however, often very difficult to medically control the blood pressure in these children. They are often treated with six or more drugs without achieving an acceptable blood pressure and the use of ACEi and ARBs becomes warranted [3]. When doing so the kidney function needs to be closely monitored both with serum creatinine and DMSA scintigraphy. The parents need to be aware of the severity of the situation and that some impairment of kidney function will be a price that we, in those circumstances, need to pay to control the BP.

\section{Surgery}

Surgery is an important treatment for children with RVD, but it is used quite differently in different units. Some major centres use surgery as their first-line treatment modality [14], while others, as we do at Great Ormond Street, use it as a last resort when other treatments have failed or been proven impossible to use.

When vascular grafts are used in small children there is always a worry that the child will out-grow the graft with a need for further surgery to put in larger grafts. There is on the other hand a worry that angioplasty and putting in a stent will make later surgery very difficult or even impossible. The use of stents should always be done in a way that does not prohibit later surgery.

There is a large number of different surgical techniques available. They include grafting of the renal arteries and/or aorta with a natural or synthetic graft. In some situations the surgery is so complicated and time consuming that the kidney needs to be taken out of the child for bench surgery. These kidneys are then auto-transplanted back to the child [15].

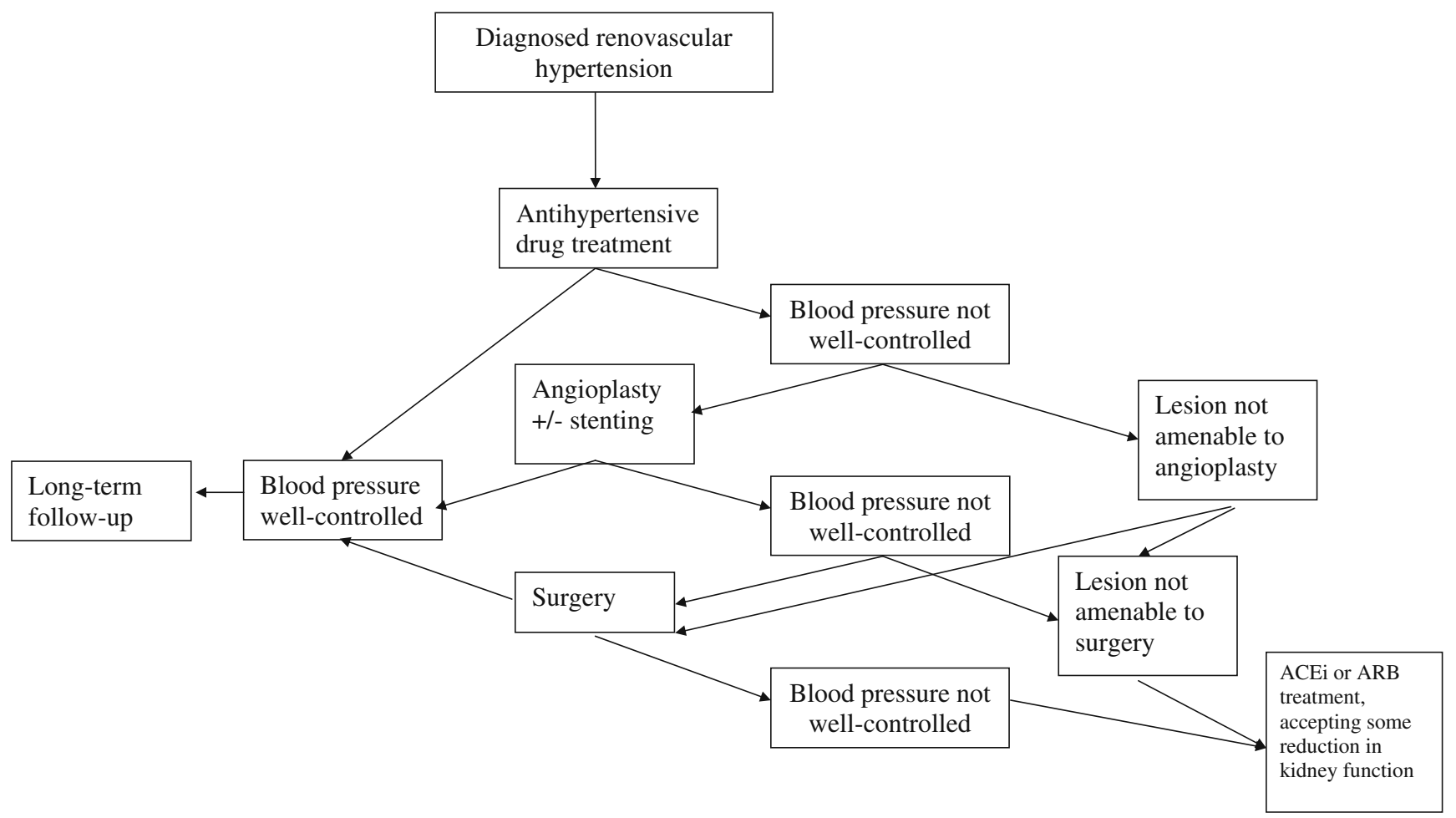

Fig. 5 Proposed treatment algorithm 
The medium long-term results of surgery are generally quite good, with at least $75 \%$ of the children having their high blood pressure cured or improved [14, 16-19].

\section{Conclusion}

Children with RVH are a challenging group both to diagnose and to treat. Formal angiography is still the gold standard investigation, as all other investigations miss a proportion of cases. We suggest that the treatment should be tailored individually using the algorithm that we propose (Fig. 5). When using this approach most children can be successfully treated both regarding their blood pressure and improved or preserved kidney function.

\section{References}

1. Dillon MJ (1997) The diagnosis of renovascular disease. Pediatr Nephrol 11:366-372

2. Tullus K, Brennan E, Hamilton G, Lord R, McLaren C, Marks S, Roebuck DJ (2008) Renovascular hypertension in children. Lancet 371:1453-1463

3. Shroff R, Roebuck D, Gordon I, Davies R, Stephens S, Marks S, Chan M, Barkovics M, McLaren C, Shah V, Dillon M, Tullus K (2006) Angioplasty for renovascular hypertension in children: 20-year experience. Pediatrics 118:268-275

4. Srinivasan A, Krishnamurthy G, Fontalvo-Herazo L, Nijs E, Keller MS, Meyers K, Kaplan B, Cahill AM (2010) Angioplasty for renal artery stenosis in pediatric patients: an 11-year retrospective experience. J Vasc Interv Radiol 21:672-680

5. Tummolo A, Marks SD, Stadermann M, Roebuck DJ, McLaren CA, Hamilton G, Dillon MJ, Tullus K (2009) Mid-aortic syndromelong-term outcome of 36 children. Pediatr Nephrol 24:2225-2232

6. Li JC, Wang L, Jiang YX, Dai Q, Cai S, Lv K, Qi ZH (2006) Evaluation of renal artery stenosis with velocity parameters of Doppler sonography. J Ultrasound Med 25:735-742
7. Abdulsamea S, Anderson P, Biassoni L, Brennan E, McLaren CA, Marks SD, Roebuck DJ, Selim S, Tullus K (2010) Pre- and postcaptopril renal scintigraphy as a screening test for renovascular hypertension in children. Pediatr Nephrol 25:317-322

8. Tullus K, Roebuck D, McLaren C, Marks SD (2010) Imaging in the evaluation of the renovascular disease. Pediatr Nephrol 25:1049-1056

9. McLaren CA, Roebuck DJ (2003) Interventional radiology for renovascular hypertension in children. Tech Vasc Interv Radiol 6:150-157

10. Cassalini E, Sfondrini MS, Fossali E (1995) Two-year clinical follow-up of children and adolescents after percutaneous transluminal angioplasty for renovascular hypertension. Invest Radiol 3:40-43

11. Tyagi S, Kaul UA, Satsangi DK, Arora R (1997) Percutaneous transluminal angioplasty for renovascular hypertension in children: initial and long-term results. Pediatrics 99:44-49

12. McTaggart SJ, Gulati S, Walker RG, Powell HR, Jones CL, Gelati S (2000) Evaluation and long-term outcome of pediatric renovascular hypertension. Pediatr Nephrol 14:1022-1029

13. Lange RA, Hillis LD (2009) Coronary revascularization in context. N Engl J Med 360:1024-1026

14. Stanley JC, Criado E, Upchurch GR Jr, Brophy PD, Cho KJ, Rectenwald JE, Michigan Pediatric Renovascular Group, Kershaw DB, Williams DM, Berguer R, Henke PK, Wakefield TW (2006) Pediatric renovascular hypertension: 132 primary and 30 secondary operations in 97 children. J Vasc Surg 44:1219-1228

15. Bleacher J, Turner ME, Quivers E, Schwartz MZ (1997) Renal autotransplantation for renovascular hypertension caused by midaortic syndrome. J Pediatr Surg 32:248-250

16. Stanley JC, Zelenock GB, Messina LM, Wakefield TW (1995) Pediatric renovascular hypertension: a thirty-year experience of operative treatment. J Vasc Surg 21:212-226

17. Chalmers RT, Dhadwal A, Deal JE, Sever PS, Wolfe JH (2000) The surgical management of renovascular hypertension in children and young adults. Eur J Vasc Endovasc Surg 19:400-405

18. Piercy KT, Hundley JC, Stafford JM, Craven TE, Nagaraj SK, Dean RH, Hansen KJ (2005) Renovascular disease in children and adolescents. J Vasc Surg 41:973-982

19. Staderman MB, Lord RH, Hamilton G, Roebuck DJ, McLaren CA, Dillon MJ, Marks SD, Tullus K (2010) Surgical treatment for renovascular hypertension in children. Nephrol Dial Transplant 25:807-813 